\title{
Zur Bestimmung der Harnsäure und Purinbasen im menschlichen Harn. ${ }^{1}$ )
}

\author{
Von \\ Martin Krüger und Julius Schmid. \\ (Der Redaktion zugegangen am 23. April 1905.)
}

Von den vier zur Ausfällung von Purinkörpern gebräuchlichen Reagentien, Phosphorwolframsäure, Kupferacetat, ammoniakalischer Silberlösung und Kupfersulfat plus Bisulfit, kommen für eine bequeme und exakte quantitative Bestimmung der genannten Verbindungen nur die beiden letztem in Betracht. Die bisher üblichsten Methoden, welche die Purinbasen neben der Harnsäure bestimmen wollen, beruhen auf der Anwendung der Silberlösung.

Als Kupferoxydulverbindungen werden Harnsäure und Basen nach dem Verfahren von Krüger und $\mathrm{Wulff}^{2}$ ) gefällt, der Niederschlag, welcher durch Kupfersulfat und Natriumbisulfit im siedenden Harn erzeugt wurde, sollte nur den Stickstoff der Purinkörper enthalten.

Diese in der ersten Zeit nach ihrer Veröffentlichung sehr häufig und von den verschiedensten Seiten angewendete Methode wurde später als unrichtig erkannt. ${ }^{3}$ ) Nach Salkowski übertrifft zwar in geringem Grade das Kupferverfahren an Schärfe das Silberreagens, aber das Plus an Purinbasenstickstoff, welches

1) Die beiden folgenden Arbeiten sind schon vor mehreren Jahren im Laboratorium der medizinischen Klinik zu Breslau (Geh. Rat Kast $\dagger$ ) ausgeführt worden. Ihre Veröffentlichung erlitt einen unerwünschten Aufschub durch die langwierige schwere Erkrankung M. Krügers; leider wurde inzwischen der allseitig beliebte Kollege und bahnbrechende Forscher auf dem Gebiet der Purinkörper der Wissenschaft $z u$ frühzeitig durch den Tod entrissen.

Schittenhelm-Schmid.

2) Diese Zeitschrift, Bd. XX, S. 176 (1895).

3) Sitzungsbericht der Frankfurter Naturforschervers. Berl. klin. Wochenschr., 1896, S. 970. Salkowski, Deutsche med. Wochenschr., 1897, S. 213 und Pflügers Arch., Bd. LXIX, S. 287 (1898).

Hoppe-Seyler's Zeitschrift f. physiol. Chemie. XLV. 
:sich nach der Krüger-Wulff schen Methode verglichen mit dem aus der Silbertällung ergibt, ist bedeutend größer, als es dem geringen Unterschiede im Fällungsvermögen der beiden Reagentien entspricht. Es müssen daher aus dem Harn noch andere N-haltige Körper mitgefällt werden. $\mathrm{Zu}$ diesen gehören nach Huppert ${ }^{1}$ ) Rhodan, Eiweiß und Albumosen, ${ }^{2}$ ) und zwar nicht nur die koagulablen, die Hellersche Probe gebenden Eiweißkörper, -sondern auch jene in jedem normalen Harn vorhandenen, von Mörner ${ }^{3}$ ) entdeckten Eiweißkörper. Huppert hat übrigens die genannten Verbindungen nicht im Kupferoxydulniederschlag des Harns nachgewiesen, sondern er führt nur an, daß diese Körper, «wenn sie dem Harn zugesetzt werden, nach dem Verfahren von Krüger - Wulff quantitativ wiedergefunden werden.»

Eine bemerkenswerte Angabe darüber, daß die Fällung der Purinkörper durch das Kupferreagens bei Anwesenheit eines bestimmten Körpers beeinträchtigt werden kann, hat Zülzer ${ }^{4}$ ) gemacht.

Wenn man nach ihm dem Harne Chlornatrium zugibt, .so sinkt der nach der Krüger-Wulffschen Methode ermittelte Stickstoff mit Zunahme des Kochsalzgehaltes. Als Erklärung fügt er die bekannte Tatsache an, daß « auch ein geringer Zusatz von Kochsalzlösung zu einer einfachen Kupfersulfatnatriumbisulfitmischung den sonst in der Hitze entstehenden Kupferoxydulniederschlag $\mathrm{zu}$ hindern imstande ist.

Nach Krüger sind die Verbindungen, welche aus Purinkörpern durch Fällung mit Kupfersulfat und Bisulfit entstehen, nicht als reine Kupferoxydulverbindungen, sondern, da sie gleichzeitig noch Schwefelsäure enthalten, welche selbst durch andauerndes Waschen nicht entfernt werden kann, als basisch schwefelsaure Purinkupferoxydulverbindungen anzusehen. Bei Gegenwart von Kochsalz scheidet sich, wie Zülzer ganz richtig erwähnt, kein Kupferoxydul aus, es entsteht vielmehr das in

1) Diese Zeitschrift, Bd. XXII, S. 556 (1896/97).

2) S. auch Strauss, Frankf. Naturforschervers., Berl. klin. Wochenschrift, 1896, S. 970.

s) Skandinav. Arch. f. Phys., Bd. VI, S. 332 (1895).

4) Berlin. klin. Wochenschr., 1896, S. 74. 
der Wärme lösliche Kupferchlorür. Dieses wird mit Purinlörpern analog der Schwefelsäure basisch salzsaure Verbindungen bilden, welche jedenfalls leichter löslich sind, als die basisch schwefelsauren Salze und daher zunächst teilweisé gelöst bleiben; lierfür spricht die Beobachtung von Zülzer. Die Löslichkeit aller basischen Salze nimmt nun aber ab mit der Zunahme ihrer Basicität und Abnahme ihrer Acidität. Dieser Übergang in schwerer lösliche, resp. unlösliche Verbindungen wird einmal bewirkt durch längeres Kochen mit Wasser oder aber schneller and glatter durch Umsetzen mit Salzen schwacher, etwa organischer Säuren, welche keine basischen Salze mehr bilden oder deren basische Salze durch Wasser sofort in Base und freie Säure gespalten werden.

Zur Entscheidung der Frage, welches von den beiden Fällungsmitteln der Purinkörper, ammoniakalische Silberlösung oder Kupfersulfat plus Bisulfit, besser zur Abscheidung und quantitativen Bestimmung derselben geeignet ist oder ob beide in gleich guter Weise diesen Zweck erfüllen, kann nach unserer Ansicht eine eingehende Prüfung der Harnsäure und sämtlicher im Harn gefundenen Purinbasen auf ihr Verhalten zu den beiden Reagentien nicht umgangen werden.

Daß Harnsäure als Magnesiumsilberdoppelsalz völlig ausfällt, ist erwiesen. Bis zu welchem Grade von Genauigkeit aber die Silberfällung bei den Purinbasen führt, ist bisher nicht systematisch geprüft worden. Bekannt ist, daß die Silberverbindungen aller Basen mehr oder weniger, wenn auch stets nur in geringem Maße, in Ammoniak löslich sind (s. darüber an späterer Stelle).

\section{Prüfung des Kupferreagens als quantitatives Fällungsmittel.}

Wir haben zunächst das Kupferreagens einer eingehenden Prüfung unterzogen, und zwar bei Harnsäure und allen Basen, welche nach den bisherigen Beobachtungen im Harne vorkommen sollen. Von den Basen standen uns mit Ausnahme des Carnins alle in chemisch reinem Zustande zur Verfügung: Xanthin, 1-Methylxanthin, 7-Methylxanthin (Heteroxanthin), 1,7Dimethylxanthin (Paraxanthin), Guanin, 7-Methylguanin (Epi- 
guanin), Adenin und Hypoxanthin. Auch Theophyllin (1,3-Dimethylxanthin) wurde noch hinzugenommen, da es bei Hunden als Stoffwechselprodukt nach Caffein auftritt.

Die Untersuchung geschah in folgender Weise: Es wurden in der Regel 0,3 $\mathrm{g}$ der freien Basen - vom Guanin kam das Sulfat und vom Hypoxanthin das Nitrat zur Anwendung in $300 \mathrm{ccm}$ Wasser, ev. unter Zusatz von etwas Natronlauge gelöst; dasselbe geschah mit der Harnsäure.

In je $50 \mathrm{ccm}$ der einzelnen Lösungen wurde dann zunächst der Stickstoff nach Kjeldahl ermittelt. In weiteren $50 \mathrm{ccm}$ wurden nach dem Verdünnen auf $100 \mathrm{ccm}$ die Basen, resp. die Harnsäure durch Kupfersulfat und Natriumbisulfit ausgefällt. $\mathrm{Zu}$ dem Zwecke wurden die Lösungen zunächst zum Sieden erhitzt und dann $10 \mathrm{ccm}$ Natriumbisulfit und $10 \mathrm{cem}$ einer 10\% igen Kupfersulfatlösung hinzugegeben. Nach dem Zusatz des Fällungsmittels wurde die Flüssigkeit noch mindestens 3 Minuten im Sieden erhalten. Der Niederschlag muß, wenn die Fällung vollständig sein soll, stets braun bis dunkelbraun gefärbt sein. Er wird dann sofort von der heißen Flüssigkeit durch ein Faltenfilter aus dem schwedischen Papier von J. Munktell abfiltriert, dreimal mit heißem Wasser ausgewaschen und samt dem Filter nach Kjeldahl zerstört. $\mathrm{Zu}$ einer dritten Portion von $50 \mathrm{ccm}$ (verdünnt auf $100 \mathrm{ccm}$ ) wurden, um den Einfluß des Natriumchlorids auf die Fällung kennen zu lernen, $2 \mathrm{~g}$ desselben hinzugegeben, zu einer vierten Portion von $50 \mathrm{ccm}$ (gleichfalls verdünnt auf $100 \mathrm{ccm}$ ) außer $2 \mathrm{~g}$ Natriumchlorid noch $6 \mathrm{~g}$ Natriumacetat. Dann wurden in beiden Versuchen in der oben angegebenen Weise wiederum die Harnsäure bezw. die Basen durch das Kupferreagens gefällt.

Die Resultate sind in der folgenden Tabelle mitgeteilt.

Von Harnsäure wurden ferner $0,0643 \mathrm{~g}$ bei Gegenwart von $2 \mathrm{~g}$ Kochsalz mit Kupfersulfat und Bisulfit gefällt. Verbraucht wurden für den N-Gehalt des Niederschlags $15,4 \mathrm{ccm}^{1 / 10} \mathrm{~N}-\mathrm{Säure}$, entsprechend 0,0647 g Harnsäure. Mit Epiguanin wurde nur ein einziger Versuch angestellt; und zwar wurden $0,0334 \mathrm{~g}$ gleichfalls aus kochsalzhaltiger Lösung gefällt. Da 10,1 ccm $1 / 10 \mathrm{~N}$-Säure verbraucht wurden; sind 0,0333 g Epiguanin wiedergefunden. 
Tabelle I.

$\mathrm{N}$-Gehalt in Kubikzentimeter 1/10 N-Säure.

\begin{tabular}{l|c|c|c|c}
\hline \hline Purinkörper & $\begin{array}{c}\text { Nach } \\
\text { Kjeld ahl } \\
\text { ccm }\end{array}$ & $\begin{array}{c}\mathrm{CuSO}_{4}+ \\
\mathrm{NaHSO}_{3} \\
\mathrm{ccm}\end{array}$ & $2 \mathrm{~g} \mathrm{NaCl}$ & $\begin{array}{c}2 \mathrm{~g} \mathrm{NaCl}+6 \mathrm{~g} \\
\text { Acetat } \\
\mathrm{ccm}\end{array}$ \\
\hline Harnsäure . . . . & 12,04 & 12,05 & - & $12,11,12,00,12,05$ \\
Xanthin . . . . . & 13,23 & 13,34 & $1: 3,20$ & 13,14 \\
1-Methylxanthin. . & 6,58 & 6,55 & 6,54 & 6,64 \\
7-Methylxanthin . . & 12,44 & 12,48 & 12,30 & 12,45 \\
Paraxanthin . . . & 11,88 & 11,88 & 11,75 & 11,96 \\
Theophyllin. . . . & 10,42 & 10,42 & 110,33 & 10,45 \\
Guaninsulfat . . . & 12,09 & 12,02 & 12,16 & 12,13 \\
Hypoxanthinnitrat. & - & 9,51 & 9,62 & 9,70 \\
Adenin . . . . & 18,14 & 17,94 & $\mathbb{1 1 8 , 0 0}$ & $18,20,18,10$
\end{tabular}

Die obigen Zahlen beweisen mit Sicherheit, daß die Fällung der Harnsäure und aller Purinbasen durch Kupfersulfat und Bisulfit stets eine vollständige ist, gleichgültig ob sie in Lösungen, die nur Purinkörper allein, oder auch bei Gegenwart von Kochsalz (in der zugesetzten Menge von 2\%) und Kochsalz plus Natriumacetat ausgeführt wird. Wenn demnach zwar das Kochsalz die Vollständigkeit der Fällung nicht hindert, so ist doch ein Einfluß desselben unverkennbar vorhanden, insofern es die Bildung des Kupferoxydulniederschlages verzögert. Eine solche Verzögerung trat am auffallendsten beim Paraxanthin ein, bei welchem die Füllung zunächst überhaupt ausblieb, bezw. nur eine geringe Trübung entstand. Aufkochen vermehrt den Niederschlag zusehends und nach 3 Minuten langem Sieden ist derselbe braun; das Filtrat scheidet beim Abkühlen keine flockige weiße Kupferoxydulverbindung mehr aus. Beim Paraxanthin übrigens erweist sich die Kupferoxydulverbindung unter dem Mikroskope als aus sehr feinen, langen Nadeln bestehend. Die durch Kochsalz verursachte Verzögerung der Purinkörperfällung wird, wie erwartet wurde, durch Natriumacetat sofort aufgehoben.

Wie verhalten sich nun die Purimbasen gegenüber der 
ammoniakalischen Silberlösung und in wie hohem Grade wird die Silberfällung durch den Gehalt der Lösungen an Ammoniak beeinflußt? Da die lösende Wirkung des Ammoniaks nach den bisherigen Angaben zwar bemerkbar, doch stets nur gering sein soll, so empfahl es sich, für die Versuche solche Basen zu wählen, deren Silberverbindungen voraussichtlich am leichtesten löslich sein müßten; es sind das die beiden Dimethylxanthine, Theophyllin und Paraxanthin - Theobromin, als drittes Dimethylxanthin, wird bekanntlich weder durch ammoniakalische Silberlösung noch durch Kupfersulfat und Bisulfit gefällt welche in ihrem Molekül nur noch eine substituierbare Imidgruppe enthalten und daher auch nur mit je einem Atom Silber Verbindungen liefern können.

Wir haben unsere Versuche zunächst mit dem Paraxanthin angestellt. Nachdem in einem gemessenen Teil einer Paraxanthinlösung der Stickstoffgehalt nach Kjeldahl bestimmt war, wurden gleiche Mengen derselben Lösung mit je $2 \mathrm{~g}$ Kochsalz, $10 \mathrm{ccm}$ Silberlösung und darauf mit wechselnden Mengen von Ammoniak versetzt. Die Silberfällung wurde nach 3 stündigem Stehen abfiltriert, mit destilliertem Wasser gewaschen und durch Aufkochen mit Magnesia usta von den letzten Resten des Ammoniak befreit. Schließlich wurde wieder der Stickstoff nach Kjeldah] ermittelt. Vorhergehende Kontrollversuche mit mehreren Basen, Paraxanthin, Adenin, Hypoxanthin, Guanin und Xanthin, hatten uns überzeugt, daß deren Silberverbindungen mit Magnesia usta in wässeriger Lösung absolut beständig sind und keine Spur von Ammoniak liefern.

Paraxanthinlösung:

1. $50 \mathrm{ccm}$ nach Kjeldahl : $8,3 \mathrm{ccm} 1 / 10 \mathrm{~N}$-Säure

2. $50 \mathrm{ccm}+2 \mathrm{~g} \mathrm{NaCl}+10 \mathrm{ccm} \mathrm{AgNO}_{3}$-Lösung +

$20 \mathrm{ccm} 10 \%$ iges $\mathrm{NH}_{3}: 5,8 \mathrm{ccm}$

3. $50 \mathrm{ccm}+2 \mathrm{~g} \mathrm{NaCl}+10 \mathrm{ccm} \mathrm{AgNO}_{3}$-Lösung +

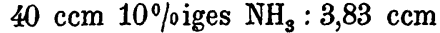

4. $50 \mathrm{ccm}+2 \mathrm{~g} \mathrm{NaCl}+10 \mathrm{ccm} \mathrm{AgNO}_{8}$-Lösung +

$60 \mathrm{ccm} \mathrm{10 \%}$ iges $\mathrm{NH}_{3}: 0,51 \mathrm{ccm}$

Bei Versuch 4. war zunächst gar kein Niederschlag entstanden, und selbst nach 3 stündigem Stehen hatte sich nur auf 
der Oberfläche, offenbar infolge Verdunsten des Ammoniaks, eine häutige Ausscheidung gebildet.

Diese Versuche konstatieren eine so große lösende Wirkung des Ammoniaks auf Paraxanthinsilber, wie sie nicht erwartet werden konnte. Es soll zugegeben werden, daß man bei Fällungen mit Silbernitrat nicht bis $\mathrm{zu}$ so hohen Prozenten an Ammoniak geht - bei Versuch 4) 5\% - daß bei Anwesenheit noch anderer Purinkörper die Fällung des Paraxanthins eine vollständigere ist, daß endlich die nicht methylierten Purine mit größerer Anzahl substituierbarer Imidgruppen schwerer lösliche Silbersalze geben, jedenfalls aber bleibt der Einfluß des Ammoniaks bestehen. Es ist daher weiterhin nötig, die sämtlichen Purinkörper, welche physiologisches Interesse beanspruchen, quantitativ auf die Löslichkeit ihrer Silbersalze in Ammoniak zu prüfen.

\section{Bestimmung der Harnsäure im Harn.}

Schon in seiner zweiten Mitteilung ${ }^{1}$ ) über das Verhalten der Purinkörper zu Kupfersulfat und Bisulfit hat M. Krüger durch mehrere Versuche bewiesen, daß die Harnsäure im Harne ebensogut mit dem genannten Reagens als mit ammoniakalischer Silberlösung nach Salkowski-Ludwig bestimmt werden kann. Die Isolierung der Harnsäure aus dem Kupferoxydulniederschlage geschah in derselben Weise wie beim Silberniederschlage durch Zersetzen mit Natriumsulfid und nachheriges Ansäuern mit Salzsäure. Die Menge der nach mehrstündigem Stehen aus der eingeengten Flüssigkeit ausgeschiedenen Harnsäure wurde aus dem nach Kjeldahl ermittelten Stickstoffgebalte berechnet. Gefunden wurden damals:

\begin{tabular}{|c|c|c|c|c|}
\hline & & $\begin{array}{c}\text { Nach } \\
\text { Salkowski-Ludwig }\end{array}$ & Nach Krüger & Differenz \\
\hline $100 \mathrm{cc}$ & cm Harn & 0,0508 g Harnsäure & 0,0504 g Harnsäure & $0,4 \mathrm{mg}$ \\
\hline 100 & $\diamond$ & 0,0442 & 0,0436 & 0,6 , \\
\hline 100 & , & 0,0370 ? & 0,0369 , & 0,1 \\
\hline
\end{tabular}

1) Diese Zeitschrift, Bd. XX, S. 172 (1895).

2) In der ursprünglichen Mitteilung befindet sich an dieser Stelle: ein Druckfehler $\star 0,0426$ g» Harnsäure. 
Die Differenz zwischen den Resultaten beider Methoden betrug daher in drei Versuchen weniger wie $1 \mathrm{mg}$. Da gemäß der ursprünglichen Vorschrift der Kupferniederschlag des Harnes erst nach zweistündigem Stehen abfiltriert werden sollte, so bot die neue Methode nur insofern einen Vorteil, als das im Vergleich $\mathrm{zu}$ der voluminösen Silberfällung geringe flockige Kupferpräzipitat leichter abfiltriert und ausgewaschen werden konnte. Nachdem sich aber die Vollständigkeit der Harnsäureund Purinbasenfällung als unabhängig von der Dauer des Erkaltens und nur abhängig von der Zeit des Erwärmens erwiesen hat - auch der Zusatz des Chlorbaryums ist als unnötig befunden -, gewährt die Kupfermethode außer dem genannten Vorteil noch den einer wesentlichen Zeitersparnis.

Es wurden noch eine Reihe weiterer Analysen ausgeführt, um die Übereinstimmung in den Resultaten beider Methoden auch in den Fällen zu zeigen, wo die Kupferniederschläge sofort nach dem Erhitzen abfiltriert werden.

\begin{tabular}{|c|c|c|}
\hline & Kupferfällung & Silberfällung \\
\hline $\begin{array}{c}\text { Harnsäure } \\
\text { in } 100 \mathrm{ccm} \text { Harn }\end{array}$ & 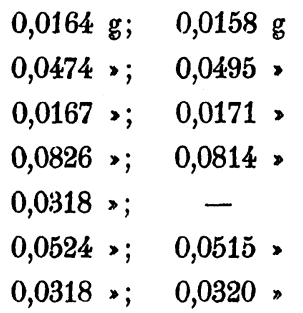 & $\begin{array}{l}0,0158 \mathrm{~g} \\
0,0492 \text {. } \\
0,0169 \text {. } \\
0,0818 \text { » } \\
0,0316 \text {. } \\
0,0517 \text {. } \\
0,0286 \text {. }\end{array}$ \\
\hline
\end{tabular}

III. Bestimmung der Purinbasen neben Harnsäure.

M. Krüger und P. Schmidt') haben sich bei ihren Fütterungsversuchen mit Purinkörpern an Kaninchen und Hunden zur Isolierung der entstandenen Stoffwechselprodukte aus dem Harne des folgenden Verfahrens bedient: Der Harn wurde siedend heiß mit Kupfersulfat und Bisulfit gefällt und der Niederschlag mit Natriumsulfid zersetzt. Aus dem mit Schwefel-

i) Ber. d. Deutsch. chem. Ges., Bd. XXXII, S. 2681. 
säure angesäuerten und eingeengten Filtrate fiel beim Erkalten lie Hauptmenge der etwa vorhandenen Harnsäure heraus, der Rest wurde in essigsaurer Lösung durch Braunstein oxydiert. Nachdem das gelöste Mangan durch Ammoniak und Ammonrarbonat entfernt war, wurden dann in der mit Schwefelsäure neutralisierten Lösung die Basen wiederum mit Kupfersulfat and Bisulfit niedergeschlagen.

In dieser zweiten Kupferfällung sind niemals andere Körper als Purinbasen allein gefunden, sodaß die Trennung derselben zeine Schwierigkeiten machte. Dasselbe gilt für einen von uns am Menschen mit Theobromin gemachten Versuch. ${ }^{1}$ ) Hiernach muß man annehmen, daß durch die Behandlung des ersten Kupferniederschlages mit Natriumsulfid, durch Eindampfen mit Mineralsäuren und endlich durch Oxydation mit Braunstein die zuerst beigemengten, stickstoffhaltigen, aber nicht zur Puringruppe gehörigen Verbindungen zerstört oder wenigstens so weit verändert werden, daß sie nicht mehr mit Kupferoxydul reagieren.

Wie groß ist der Unterschied im Stickstoffgehalte der ersten und zweiten Kupferfällung? Hierüber gibt folgender Versuch Aufschluß: In $100 \mathrm{ccm}$ Harn wurde der angebliche Purinkörper-N nach Krüger-Wulff bestimmt. Dann wurde der Harnsäuregehalt von $300 \mathrm{ccm}$ desselben Harnes mit dem Kupferverfahren ermittelt. Das Filtrat von der Harnsäure $(75 \mathrm{ccm})$ wurde zur Beseitigung des Restes an Harnsäure mit Braunstein in essigsaurer Lösung oxydiert und dann mit Kupfersulfat und Bisulfid behandelt. Der Stickstoff dieses Niederschlages gibt den Purinbasen-Stickstoff an. Gefunden wurden, ausgedrückt in Kubikzentimetern ${ }^{1 / 10} \mathrm{~N}$-Säure, folgende Zahlen:

1. Nach Krüger-Wulff in 100 Harn $22,61 \mathrm{ccm}$; in $300 \mathrm{ccm}$ demnach $67,83 \mathrm{ccm}$.

2. Harnsäure in 300 Harn $52,37 \mathrm{ccm}$; mit Hinzurechnung der Korrektur für in $75 \mathrm{ccm}$ Filtrat in Lösung gebliebene Harnsäure 53,27 ccm Basenstickstoff, im Filtrate von der Harnsäure $=4,29 \mathrm{ccm}$. Nach Krüger-Wulff sind in 300 Harn Basenstickstoff gefunden 67,83-53,27=14,0̄6 ccm, im

1) Diese Zeitschr., Bd. XXXII, S. 104 (1901). 
Filtrate von der Harnsäure nur 4,29 $\mathrm{ccm}=\mathbf{2 9 , 5} \%$ vom ersteren Wert. Demnach sind 70,5\% des nach Krüger-Wulff ermittelten Stickstoffes bei den mit dem ersten Kupferniederschlage vorgenommenen Manipulationen zerstört worden.

Gibt nun die zweite Kupferfällung den richtigen Purinbasen-Stickstoff an, oder enthält sie immer noch fremde stickstoffhaltige Beimengungen, oder gibt sie zu niedere Werte an? Letzteres würde dafür sprechen, daß die Purinbasen selbst auf dem Wege bis zur zweiten Kupferfällung zum Teil zerstört werden.

Wie verhalten sich die Purinbasen gegen Säuren, Alkali und Braunstein? Durch Säuren wird der Stickstoff aller Purinkörper vollständig als Ammoniak und Amidosäuren abgespalten; jedoch geschieht dies nur durch starke Säuren, wie konzentrierte Salzsäure oder konzentrierte Schwefelsäure, welche mit dem doppelten Volumen Wasser verdünnt ist, und bei mehrstündigem Erhitzen unter Druck auf Temperaturen, welche weit über $100^{\circ}$ liegen. Beim Eindampfen auf dem Wasserbade dagegen und bei Anwesenheit so geringer Mengen freier Säuren, wie im vorliegenden Falle ist eine Zerstörung der Basen völlig ausgeschlossen.

Die Einwirkung von Alkalien auf die Purinkörper hat E. Fischer einer eingehenden und systematischen Untersuchung unterzogen und gefunden, daß ihre Beständigkeit mit Zunahme der Methylgruppen abnimmt. ${ }^{1}$ ) Von den im Harn vorkommenden Purinbasen wird demnach das Paraxanthin am meisten unter dem Alkali zu leiden haben; von 0,4935 g dieser Base wurden bei 15 Stunden langem Erhitzen auf $100^{\circ}$ mit $10 \mathrm{ccm}$ N-Kalilauge $32 \%$ zerstört. Diese anscheinend sehr beträchtliche Wirkung des Alkali auf das Paraxanthin ist zweifellos proportional der Länge der Zeit und abhängig von der Konzentration des Alkali.

Da nun die Umsetzung des Kupferniederschlages nach der Vorschrift in einer Lösung erfolgt, welche höchstens $0,15-0,20 \%$ Natriumsulfid enthält, und dieselbe nur einen Zeitraum von etwa 1 Minute erfordert, so ist klar, daß eine

1) Ber. d. Deutsch. chem. Ges., Bd. XXXII, S. 500 (1899). 
Zerstörung auch nur eines geringen Teiles der Basen hierbei nicht erfolgen kann, wie auch folgende Versuche beweisen.

In je $200 \mathrm{ccm}$ Harn wurden die Harnsäure und Basen durch Kupfersulfat und Bisulfit gefällt; dann wurden die ausgewaschenen Niederschläge einmal durch Schwefelwasserstoffgas in saurer Lösung, der andere durch Natriumsulfid zersetzt. Beide Filtrate wurden bei saurer Reaktion eingedampft und nach Entfernung der Harnsäure in der oben angedeuteten Weise die Basen wiederum durch Kupfersulfat und Bisulfit gefällt. Die in der Tabelle angegebenen Zahlen bedeuten die Anzahl der nach Kjeldahl verbrauchten Kubikzentimeter 1/10-Normalsäure.

\begin{tabular}{clc} 
Harn & \multicolumn{1}{c}{$\mathrm{H}_{2} \mathrm{~S}$} & $\mathrm{Na}_{2} \mathrm{~S}$ \\
1. & $3,75 \mathrm{ccm}$ & $3,69 \mathrm{ccm}$ \\
2. & $4,13$. & $4,29$.
\end{tabular}

Daß endlich auch die Harnsäure selbst trotz häufiger gegenteiliger Behauptungen nicht beim Erhitzen mit Natriumsulfid zersetzt wird, beweist die festgestellte Übereinstimmung in den Resultaten der Salkowskischen und der Ludwigschen Methoden.

Es bleibt noch übrig, den Einfluß des Braunsteins auf die Purinbasen zu ermitteln. Daß Harnsäure beim Erhitzen mit Braunstein in essigsaurer Lösung vollständig in das durch Kupfersulfat + Bisulfit nicht mehr fällbare Allantoin übergeführt werden kann, daß ferner Adenin bei gleicher Behandlung nicht angegriffen wird, somit eine quantitative Bestimmung des Adenins neben Harnsäure möglich ist, ist schon früher bewiesen worden. ${ }^{1}$ ) Die übrigen Purinbasen erweisen sich nun in gleichem Maße widerstandsfähig gegenüber der Oxydation mit Braunstein.

Vor den diesbezüglichen Resultaten mögen die Versuche erwähnt werden, durch welche festgestellt wurde, welcher Zeitraum der Erhitzung und welche Temperatur zur Zerstörung der geringen bei unserer Methode in Betracht kommenden Harnsäuremenge genügt.

Es wurden je $10 \mathrm{mg}$ Harnsäure in $100 \mathrm{ccm} \mathrm{H}_{2} \mathrm{O}$ mit 1-3 ccm 10\% \%iger Essigsäure und $10 \mathrm{ccm}$ einer Aufschwemmung von $\mathrm{MnO}_{2}$ in Wasser (deren Herstellung s. Methode) zum Sieden

1) Diese Zeitschrift, Bd. XXI, S. 317 (1896). 
erhitzt. Nach Beendigung der Oxydation wurde der etwa vorhandene Rest der Harnsäure durch Kupfersulfat und Bisulfit ausgefällt. Es zeigte sich nun, daß in diesem Filterrückstand niemals $\mathrm{N}$ nachzuweisen, somit also die Harnsäure stets vollständig in Allantoin übergeführt war, gleichgültig ob ihre Lösung mit $\mathrm{MnO}_{2} 3$ oder 1 Minute im Sieden erhalten wurde oder ob nach dem Erhitzen bis zum Siedepunkt die Flamme entfernt wurde und nunmehr der Braunstein eine Minute lang einwirkte.

Von Basen wurde neben Xanthin Heteroxanthin und Paraxanthin, von denen der geringste Widerstand gegen oxydierende Mittel vorausgesetzt werden konnte, der Einwirkung des $\mathrm{MnO}_{2}$ unterworfen. Ihre Lösungen, deren Gehalt an Basen-N durch eine $\mathrm{Cu}-\mathrm{F}$ ällung bestimmt wurde, wurden gleichfalls mit $1-3 \mathrm{ccm}$ Essigsäure und $10 \mathrm{ccm}$ Braunsteinlösung eine Minute lang in lebhaftem Sieden erhalten und dann mit Kupfersulfat und Natriumbisulfit gefällt. Die Zahlen der Tabelle geben den N-Gehalt der Fällung in ${ }^{1 / 10}$ N-Säure an.

\begin{tabular}{lcc}
\multicolumn{1}{c}{ Base } & Cu-Fällung vor der & $\mathrm{nach} \mathrm{Oxydation} \mathrm{mit}$ \\
& Oxydation & $\mathrm{MnO}_{\mathbf{8}}(1 \mathrm{Min})$. \\
Xanthin & $5,29 \mathrm{ccm}$ & 5,$36 ; 5,20 ; 5,31 \mathrm{~cm}$ \\
Heteroxanthin & $4,97:$ & $4,69 \mathrm{~cm}$ \\
Paraxanthin & $4,52:$ & $4,45:$ \\
\multicolumn{1}{c}{} & 9,44 & 9,38
\end{tabular}

Die Abweichungen beider Zahlenreihen liegen innerhalb der Versuchsfehlergrenzen.

Vergleich der Kupfer- und Silberfällung.

1. $600 \mathrm{ccm}$ Harn mit Kupfersulfat und Natriumbisulfit gefällt; dann mit Natriumsulfid zersetzt und eingedampft. Filtrat mit $\mathrm{NH}_{3}$ alkalisch, mit Essigsäure schwach sauer gemacht und mit $\mathrm{MnO}_{2}$ oxydiert; mit Natriumkarbonat und Natronlauge versetzt und auf 200 verdünnt, davon 2 mal je $100 \mathrm{ccm}$ abfiltriert.

a) mit $\mathrm{CuSO}_{4}+$ Bisulfit gefällt, verbraucht $4,65 \mathrm{ccm}$

b) mit ammoniakalischer Silberlösung gefällt, verbraucht $4,75 \mathrm{ccm}$.

2. dieselbe Versuchsanordnung:

a) mit $\mathrm{CuSO}_{4}+\mathrm{Bisulfit}$ gefällt, verbraucht $7,89 \mathrm{ccm}$

b) mit ammoniakalischer Silberlösung gefällt, verbraucht $\mathbf{7 , 7 0} \mathrm{ccm}$. 
3. je 600 ccm Harn:

a) Fällung mit $\mathrm{CuSO}_{4}+$ Bisulfit (wie o.). Die Fällung der Basen mit $\mathrm{CuSO}_{4}+$ Bisulfit: verbraucht $4,95 \mathrm{ccm}$.

Fällung der Basen mit $\mathrm{Ag}_{2} \mathrm{O}$ : verbraucht $\mathbf{5 , 0 0} \mathrm{ccm}$.

b) Fällung mit ammoniakalischer Silberlösung. Die Fällung der Basen mit $\mathrm{CuSO}_{4}+$ Bisulfit: verbraucht $5,05 \mathrm{ccm}$.

Fällung der Basen mit $\mathrm{Ag}_{2} \mathrm{O}$ : verbraucht 4,86 ccm.

4. je $600 \mathrm{ccm}$ Harn:

a) Fällung mit $\mathrm{CuSO}_{4}+$ Bisulfit. Fällung der Basen mit $\mathrm{Ag}_{2}$ 0-Lösung: verbraucht $4,72 \mathrm{ccm}$.

Fällung der Basen mit $\mathrm{CuSO}_{4}$ : verbraucht $4,88 \mathrm{ccm}$.

b) Fällung mit ammoniakalischer Silberlösung. Fällung der Basen mit $\mathrm{Ag}_{2} \mathrm{O}-\mathrm{L}$ ösung: verbraucht $\mathbf{4 , 5 5} \mathrm{ccm}$.

Fällung der Basen mit $\mathrm{CuSO}_{4}$ : verbraucht $4,69 \mathrm{ccm}$.

Die Analysen, denen noch mehrere mit gleichem Resultat angereiht werden könnten, ergeben, daß jede Kombination beider Methoden ohne Beeinträchtigung des Resultates möglich ist.

Damit glauben wir genügend Beweise erbracht zu haben für die Sicherheit, mit der das Kupferverfahren eine vollständige Ausfällung der Purinkörper verbürgt. Es gebühri dem Kupferreagens, welches durch die mißlungene Krüger-Wulffsche Methode in Mißkredit gekommen war, ein ebenbürtiger Platz neben dem Silberreagens. Diesem gegenüber ist es sogar überlegen in der Schnelligkeit, mit der die Niederschläge entstehen und weiter verarbeitet werden können.

$\mathrm{Da}$ die Ausführung unserer Methode bereits durch die Liebenswürdigkeit des Herrn Prof. Thierfelder in dem von ihm neubearbeiteten Hoppe-Seylerschen Lehrbuch Aufnahme fand, glauben wir an dieser Stelle auf eine detaillierte Wiedergabe verzichten $z u$ dürfen. 